\section{Micromanaging latent herpes}

\section{By Michael J. Haas, Senior Writer}

A report in Nature provides the first biological evidence that two viral microRNAs contribute to latency of herpes simplex virus- 1 by blocking the expression of viral proteins thought to regulate viral reactivation and active infection. ${ }^{1}$ The findings could lead to the development of agents that reactivate latent virus so the infection can be cured with therapeutics targeting active virus. Current therapies treat active infections but cannot prevent the reactivation of latent virus that is responsible for recurring outbreaks.

Company representatives contacted by SciBX said the findings advance the understanding of the mechanisms of latency in herpes simplex virus-1 (HSV-1) infection and might lead to a similar understanding of other viruses in the herpes family. They also concurred that developing an antisense therapeutic may be the preferred strategy, but differed over whether the goal should be to reactivate HSV-1 or keep it latent.

The Nature study resulted from a collaboration between Duke University Medical Center and Harvard Medical School and was headed by Bryan Cullen, professor of genetics and microbiology at Duke, and Donald Coen, professor of biological chemistry and molecular pharmacology at Harvard.

"In principle, if you could induce reactivation of the virus, then it could be treated with acyclovir or a next-generation compound like valacyclovir that inactivates replicating virus," Coen told SciBX. "You could potentially cure the viral infection."

Acyclovir is a generic used to treat HSV infection and varicella zoster virus (VZV) infection. VZV (herpes zoster) causes chicken pox and shingles. Valtrex valacyclovir from GlaxoSmithKline plc is approved in the U.S. to treat and suppress cold sores and genital herpes caused by HSV infection and to treat herpes zoster.

\section{How HSV lies low}

Although the functions of most viral miRNAs remain unknown, a growing body of evidence suggests they can regulate viral functions, including latency, in infected cells. ${ }^{2}$ Computational and bioinformatics studies have led to the hypothesis that herpes viruses use viral miRNAs to enter and maintain latency. ${ }^{3-6}$ But biological evidence that specifically linked HSV-1 latency to viral miRNA was lacking, in part because latency of that virus occurs only in nondividing, terminally differentiated neurons.

"As they are nondividing, these neurons can only be cultured for a short time ex vivo," Cullen said.
Studying latent HSV-1 infection presents another issue, he said. "Only about $10 \%$ of cells in a ganglion are neurons, and only about $1 \%$ of these become latently infected. Our novel approach got us around the problem of only a small number of cells being infected in vivo."

The research team began by cloning miRNAs from mouse neural ganglia that had been latently infected with HSV-1. Sequencing of these ex vivo ganglia identified five highly expressed viral miRNAs.

Four of them-miRNA-H2-3p, miRNA-H3, miRNA-H4 and miRNA-H5-were produced from latency-associated transcript (LAT), a viral transcription factor thought to regulate expression of HSV-1 genes during latent infection. Two earlier research teams-one led by Coen, the other at The Rockefeller University-had between them predicted three of these four miRNAs by computational analysis. ${ }^{7,8}$

The fifth miRNA identified by the Cullen-Coen team, miRNA-H6, was produced from an unknown transcript and was not predicted in either of the two earlier studies.

Next, the team showed in vitro that miRNA-H2-3p and miRNA-H6 downregulated the respective expression of two viral proteins: human herpes virus-infected cell polypeptide 0 (ICP0) and ICP4. These proteins are thought to play a role in the early stages of viral activation and productive (active) infection, and thus are also referred to as HSV-1 immediate-early regulatory proteins. Inhibition of the two miRNAs with small hairpin RNA restored the expression of each protein.

Taken together, the results provide the first biological evidence that HSV-1 maintains latency by using at least two viral miRNAs to downregulate proteins involved in viral reactivation. Moreover, the results explain how LAT contributes to viral latency.

"There is a body of previous literature, going back to the 1990s, showing that LAT represses productive-cycle gene expression" but not how LAT accomplishes this, Coen said. "So it was especially interesting to us that two miRNAs can repress two immediate-early regulatory proteins."

He added: "Latency can be thought of, operationally, as the repression of viral gene expression. Our study provides a potential explanation for how latency occurs-though we haven't shown that these miRNAs are the only way to induce latency."

\section{Coaxing virus out}

Company representatives contacted by SciBX agreed that the findings expand the understanding of latency in HSV-1 and could lead to new therapeutics.

"The Nature paper makes a strong case for connecting miRNAs to viral latency in HSV-1," said Jürgen Soutschek, senior director for scientific development at Regulus Therapeutics LLC. "Previous data show that CMV uses a comparable miRNA strategy to hide in cells. But the Nature paper provides additional evidence that viral miRNAs are connected to this hiding strategy for a clinically relevant virus."

Cytomegalovirus (CMV) is a widespread but typically asymptomatic infection that does not require treatment in most infected individuals.

Regulus is a joint venture between Isis Pharmaceuticals Inc. and Alnylam Pharmaceuticals Inc. with an exclusive license to both parent 
companies' miRNA technologies. Regulus also has an ongoing material transfer agreement with Cullen and his lab at Duke University Medical Center.

Soutschek noted that the Nature study overcomes one hurdle in targeting miRNAs-identifying which ones actually play a significant role in the disease.

Even when an miRNA appears to play a role in a disease, he said, there is no guarantee that targeting it will have an appreciable effect. One reason for such muted efficacy is that a given miRNA may have different mRNA targets in different tissues. Another is that an miRNA typically cannot modulate a disease-relevant mRNA that has a fivefold higher level of expression than the miRNA.

"Very often you don't know all of the targets plus their expression levels," Soutschek said. "You can't make predictions, so you just need to test the outcome of a particular miRNA modulation. It's our experience that in several cases it just doesn't work."

According to Soutschek, Regulus has developed a solution to the other problem-how to deliver an miRNA inhibitor to target cells and tissues. Regulus is developing antimiRs (antisense oligonucleotides that target miRNAs) to disrupt miRNAs' interactions with their respective mRNA targets.

Soutschek said Cullen and Coen intend to use the company's antagomirs-a subclass of antimiRs that use a cholesterol-based conjugate to deliver the antisense payload to epithelial and neural cells-in their ongoing experiments.

Regulus' lead program, which targets viral miRNA-122 to treat HCV infection, is in preclinical development.

William Marshall, president and CEO of miRagen Therapeutics Inc., agreed that targeted delivery of miRNA antagonists is an ongoing but not insurmountable challenge. But he said another complicating factor is that miRNA molecules typically regulate the expression of multiple proteins. Thus, Marshall wanted to know whether the HSV-1 miRNAs affected the expression of proteins besides ICP0 and ICP4.

"The more specific the miRNA's action, the easier it is to consider targeting it with an antisense short RNA," he said. If the viral miRNAs have targets in the host cell, inhibiting these miRNAs could have unwanted side effects.

Marshall noted this would only be a problem if the HSV-1 miRNAs share significant sequence homology with the host cell genome.

Coen said it was difficult to say what would constitute a significant homology given that miRNAs are thought to act through rather short sequences-about eight units long-that base pair with mRNA. "The HSV miRNAs can certainly be distinguished readily from host miRNAs," he said. "That said, HSV miRNAs might very well act, at least in part, by affecting cellular mRNAs."

miRagen's lead program is targeting miRNA-208, a heart-specific miRNA involved in cardiac contractility. It is in preclinical development to treat heart failure.

Kenji Sudo, a research fellow in infectious diseases at Astellas Pharma Inc., noted that LAT was already known to play an important role in the establishment and maintenance of HSV-1 latency and in viral reactivation. "However, it is very interesting that a specific entity-a
microRNA-expressed by HSV-1 itself regulates viral proteins in HSV-1 latency," he said.

Indeed, the findings could hold broader commercial potential if the mechanism of latency in HSV-1 is found to be conserved in other herpes viruses, such as HSV-2, VZV and CMV. "A well-conserved property among different viruses would be a strong candidate for antiviral agents," according to Sudo.

Astellas has ASP2151, an inhibitor of the helicase-primase complex, in a Phase II trial to treat genital herpes and herpes zoster.

Shani Waninger, associate director of R\&D at Adventrx Pharmaceuticals Inc., said the Nature paper provides the most direct confirmation to date connecting LAT to viral latency through viral miRNA. "This contributes to the overall understanding of the mechanism of latency of HSV by clarifying a key step: the mechanism of suppression of ICP4 and ICP0 expression," she said.

Waninger noted that other herpes family viruses "have also been suggested to have a similar mechanism of latency. This work should give some credence to those theories and facilitate similar studies with other viruses."

She said an interesting follow-up study would be to investigate what upstream factors regulate the expression of LAT itself. "It is likely that LAT downregulation is a key step in switching from latent infection to active infection," she said. "Triggers for this switch are important in understanding the HSV life cycle."

Adventrx's Thiovir (ANX-201) is a pyrophosphate analog reverse transcriptase inhibitor that blocks viral polymerases. It is in preclinical development as a topical therapeutic to treat HSV and an oral therapeutic to treat HIV infection.

\section{Let sleeping dogs lie?}

Beyond the issues involved in developing an antisense agent that targets HSV-1 miRNA, an open question is whether reactivating latent virus in order to cure it with therapeutics that target active virus would be the better therapeutic approach instead of keeping the virus permanently latent to prevent outbreaks of active infection.

Andrew Spaltenstein, VP of antiviral drug discovery at GlaxoSmithKline, said latency is an important factor in HSV-1 infection. "The potential for a drug that can induce the activation of the virus from its latent state, so that it can then be treated or eradicated with antiviral agents, is very significant," he said. "Eliminating the infection would be a better option than attempting to keep the virus permanently latent, as the latter would involve long-term administration of treatment."

But he noted that the Nature findings were a long way from a therapeutic for either strategy. "The microRNA field is in its infancy and will have a long way to go before it produces commercial products," he said.

Adventrx's Waninger agreed that an agent with the ability to eradicate HSV-1 would have a potentially large market because it would allow patients to remain drug- and virus-free after treatment. But she said the Nature paper does not directly demonstrate that targeting miRNAs could potentially result in a cure, and more preclinical and clinical studies are needed. 
"I think any claims of being able to eradicate virus in a patient should be made cautiously, and maybe met with skepticism, until solid evidence is presented," she said.

Waninger noted that similar claims were made at the advent of highly active antiretroviral therapy (HAART) for HIV patients. "After the initial excitement that there was a 'cure' for HIV, the reality and persistence of HIV reservoirs was realized," she said.

Waninger said a more realistic translational strategy would aim to keep HSV-1 completely latent with an miRNA agonist or mimic rather than reactivate it with an miRNA antagonist.

"The therapeutic value would lie in the ability to keep the virus dormant by delaying or preventing the switch from latency to active viral replication," she said. "If the miRNA agonist or mimic could be delivered to the infected cells, the virus should remain dormant."

Coen acknowledged that induced reactivation of a patient's reservoir of latent virus held the potential for undesirable effects, such as neural damage. "Generally people with recurring HSV-1 don't have nerve damage, but there are some reported cases," he said. "But would the potential for damage from induced reactivation be worse than the damage from recurrent outbreaks?"

Coen thought some more important issues were how efficiently the virus could be reactivated and how efficiently replicating virus could be inactivated.

"A significant question is whether you could get enough drug into the sensory ganglia to reach neurons and reactivate the virus," he said. "Some literature suggests that acyclovir doesn't reach the neurons in patients with naturally latent infections-but this could be a dosing issue."

\section{Myriad possibilities}

Both Cullen and Coen are continuing to work in the area. Coen said he plans to conduct two complementary sets of experiments that would further explore the roles of miRNA-H3-2p and miRNA-H6-and the other miRNAs - in viral latency and reactivation.

One set of experiments will use antagomirs to block the functions of the viral miRNAs, to determine whether the compounds can induce expression of productive-cycle genes or even induce active infection of the virus. The other set will examine how miRNA of mutant viruses-in which miRNA coding is altered or expression is reduced-affect viral latency.
Cullen added that both his group and Coen's plan to study the functions of the other viral miRNAs identified in the Nature study. "One miRNA is clearly not regulating viral mRNA, indicating it probably has a cellular target," he said. "All four might have other targets. We want to understand all of these regulatory functions."

Cullen's lab is also developing a tissue culture model that could bypass the need to study HSV latency in vivo. "We're building a cell line that expresses [viral] miRNAs at levels comparable to those found in vivo," he said. "This could be used to validate the antagomirs from Regulus or by anyone interested in latent viral work."

Both researchers said they might study the role of viral miRNAs in HSV-2 latency at some future date.

Harvard Medical School and Duke University Medical Center have submitted a patent application for the HSV-1 miRNAs and antisense agents that would target them.

"We are in early stage licensing discussions with Regulus, but nothing has been finalized at this point," said Cullen.

\section{REFERENCES}

1. Umbach, J. et al. Nature; published online July 2, 2008; doi:10.1038/nature07103

Contact: Bryan Cullen, Duke University Medical Center, Durham, N.C. e-mail: culle002@mc.duke.edu

2. Swaminathan, S. J. Cell Physiol. 216, 321-326 (2008)

3. Grey, F. et al. PLoS Pathog. 3, e163; published online November 2, 2007; doi:10.1371/journal.ppat.0030163

4. Barth, S. et al. Nucleic Acids Res. 36, 666-675 (2008)

5. Murphy, E. et al. Proc. Natl. Acad. Sci. USA 105, 5453-5458 (2008)

6. Yin, Q. et al. J. Virol. 82, 5292-5306 (2008)

7. Cui, C. et al. J. Virol. 80, 5499-5508 (2006)

8. Pfeffer, S. et al. Nat. Methods 2, 269-276 (2005)

\section{COMPANIES AND INSTITUTIONS MENTIONED}

Adventrx Pharmaceuticals Inc. (AMEX:ANX), San Diego, Calif. Alnylam Pharmaceuticals Inc. (NASDAQ:ALNY), Cambridge, Mass. Astellas Pharma Inc. (Tokyo:4503), Tokyo, Japan

Duke University Medical Center, Durham, N.C. GlaxoSmithKline plc (LSE:GSK; NYSE:GSK), London, U.K. Harvard Medical School, Boston, Mass. Isis Pharmaceuticals Inc. (NASDAQ:ISIS), Carlsbad, Calif. miRagen Therapeutics Inc., Boulder, Colo. Regulus Therapeutics LLC, Carlsbad, Calif. The Rockefeller University, New York, N.Y. 